\title{
EDITORIAL
}

\section{Percutaneous coronary intervention in diabetics: time to consider "intimal remodelling therapy"?}

\section{P F Ludman}

Heart 2002;88:213-215

\section{Can "intimal remodelling therapy" by percutaneous coronary intervention improve the otherwise poor prognosis of diabetic patients with severe diffuse multivessel disease?}

A n apparently inexorable sequence of remarkable developments in the techniques of percutaneous coronary intervention (PCI) have led to this method being poised to become the dominant tool for coronary revascularisation. Nevertheless, interventional cardiologists will not be able to make this claim until we can be confident about the best management strategy for patients with diabetes and multivessel coronary disease.

Diabetes is a vascular disease. Patients with both type 1 and type 2 diabetes carry the burden of a high incidence of premature fatal macrovascular disease. Type 2 diabetes, best described as a cluster of risk factors for cardiovascular disease (insulin resistance, dyslipidaemia, obesity, hypertension) is associated with a reduction in life expectancy of 8-10 years in the 40-70 year age range. ${ }^{1}$ Type 1 diabetes is rather different. It is not associated with other classical cardiovascular risk factors. Indeed the normal or low triglyceride concentrations, normal or high HDL (high density lipoprotein) cholesterol and normal LDL (low density lipoprotein) cholesterol should be protective. Nevertheless, type 1 diabetes carries the same disturbingly high rate of cardiovascular disease. The standardised mortality ratio for men and women aged 40-59 years is 4.7 and 7.8 , respectively. ${ }^{2}$ Type 2 diabetes accounts for more than $95 \%$ of all diabetes, and its prevalence is rising at an alarming rate. In 2001 it was estimated that over 150 million people in the world were diabetic. Because of a sharp rise in prevalence over the last decade, it is predicted that by 2010 the global prevalence of diabetes will be in excess of 220 million. ${ }^{3}$ Against this backdrop is a series of studies that have consistently failed to show any significant reduction in fatal cardiovascular disease with tight glycaemic control. Only aggressive blood pressure control and the use of HMG-CoA reductase inhibitors have been convincingly shown to be efficacious in slowing the progression of macrovascular disease.

Correspondence to: Dr Peter F Ludman, Cardiology Department, Queen Elizabeth Hospital, University of Birmingham, Edgbaston, Birmingham B15 2TH, UK;

p.f.ludman@bham.ac.uk

\section{BARI TRIAL}

The bypass angioplasty revascularization investigation (BARI) trial randomly assigned 1829 patients with multivessel coronary disease to a strategy of initial revascularisation by coronary artery bypass surgery (CABG) or PCI. ${ }^{4}$ Patients were recruited from 1988 to 1991 and followed for 5.4 years. No stents or glycoprotein IIb/IIIa receptor antagonists were used. The primary end point of all cause mortality was similar $(10.7 \%$ for CABG $v 13.7 \%$ for PCI, $\mathrm{p}=$ NS). In 1992 (after all patients had been recruited), the data and safety monitoring board requested an analysis of diabetic patients on the basis of published reports of adverse outcomes of PCI after thrombolytic treatment in a subgroup of patients. ${ }^{5}$ It was this prompt for a retrospective analysis of a trial never intended to address the issue of differential outcome in diabetics that first raised anxieties about the optimal revascularisation strategy for diabetic patients with multivessel disease. Treated diabetes was defined as the use of oral hypoglycaemic agents or insulin, and 19\% of the randomised population met these criteria. Unfavourable baseline characteristics appeared to be similar in the CABG and PCI groups, but there appeared to be considerably better survival at 5.4 years in those randomised to initial treatment by CABG (19.4\% $v 34.5 \%, \mathrm{p}=0.003)$. The cardiac mortality rates were $5.8 \%$ (CABG) versus $20.6 \%$ (PCI) $(\mathrm{p}=0.0003){ }^{6}$ The benefits of CABG in the diabetic cohort appeared to be confined to those patients who had received an internal mammary artery (IMA) graft. The cardiac mortality was $2.9 \%$ when at least one IMA was used, and $18.2 \%$ when only saphenous veins were used. Looking at the entire randomised population, there was a steady divergence in the survival curves, so that by seven years, a survival advantage was seen in those patients initially treated by CABG (mortality $15.6 \%$ CABG $v 19.1 \%$ PCI, $\mathrm{p}=0.043) .{ }^{7}$ All the observed treatment difference could be attributed to a substantial difference in outcome in the treated diabetic subgroup. Treated diabetics assigned CABG had a mortality of $23.6 \%$ versus $44.3 \%$ for PCI, while patients without treated diabetes had a virtually identical seven year mortality (13.6\% for CABG $v 13.2$ for PCI).

\section{OTHER RETROSPECTIVE ANALYSES}

Unfortunately there have been no prospective randomised controlled trials to try to answer the

Abbreviations: ARTS, arterial revascularisation study; BARI, bypass angioplasty revascularization investigation; CABRI, coronary angioplasty versus bypass revascularisation investigation; $C A B G$, coronory artery bypass graft; EAST, Emory angioplasty versus surgery trial; $\mathrm{HDL}$, high density lipoprotein; IMA, internal mammary artery; LDL, low density lipoprotein; $\mathrm{PCl}$, percutaneous coronary intervention; RITA, randomised intervention treatment of angina 
questions raised by BARI, but other retrospective analyses lend support to the conclusions from BARI. In the smaller Emory angioplasty versus surgery trial (EAST) there were few diabetic patients randomised. While there was no difference in survival at three years, by eight years a trend towards better survival with surgery had emerged. ${ }^{8}$ A recent analysis of the four year mortality data of the coronary angioplasty versus bypass revascularisation investigation (CABRI) also supports the unfavourable relation between multivessel angioplasty and diabetes. ${ }^{9}$ More contemporary data come from the arterial revascularisation study (ARTS) trial in which stents were used for all PCI procedures (but glycoprotein IIb/IIIa receptor antagonists were used in only 3.5\%). ${ }^{10}$ Of the 1205 patients randomised, 208 were diabetic. Follow up to only one year is currently available, but even at this early stage it is possible to see that diabetics treated by multivessel PCI with stenting had a trend to worse one year outcome than patients assigned to CABG or non-diabetics treated by PCI with stenting. ${ }^{11}$ Mortality in diabetic patients assigned to PCI and stenting was twice as high as among those assigned to undergo CABG $(6.3 \%$ v $3.1 \%, \mathrm{p}=\mathrm{NS}) .^{12}$

It is easy to dismiss these findings on the basis that they represent treatment in a different era, that the use of glycoprotein IIb/IIIa receptor antagonists and drug eluting stents to inhibit restenosis will so alter the landscape, and that these potentially unfavourable findings will be diminished. But this will only be the case if the mechanism of adverse events is appropriately targeted by these new developments. There are persuasive data to suggest that the adverse medium term outcome seen following multivessel PCI in diabetics is not caused by the effects of angioplasty on a culprit lesion, but instead can be explained by atheroma progression in other segments of the coronary artery-those that have not been dilated by an angioplasty balloon. PCI is a "spot" treatment that leaves the majority of the artery unmodified. Indeed, because of the increased risk of restenosis, there is a disincentive to dilate long segments of coronary artery. Thus a patient treated by angioplasty is still vulnerable to plaque evolution at another site in the treated artery. Provided that the risk of such atheroma progression is low (as it is in non-diabetic patients with mild disease) then PCI is a very suitable treatment. A surgical graft, however, in bypassing the proximal segments of atherosclerosis laden coronary arteries, protects against the sequelae of plaque rupture in these segments. Because it is the proximal $6 \mathrm{~cm}$ of epicardial vessels that are most prone to developing obstructive lesions, a bypass graft (particularly an internal mammary artery graft with its favourable long term patency rate) is likely to offer better protection from late occlusive events in patients at high risk of atheroma progression.

\section{"When diabetic patients with less extensive disease are studied, the outcomes of treatment by $\mathrm{PCl}$ or $\mathrm{CABG}$ are similar"}

The uncoupling of periprocedural risks from mortality caused by disease progression has been addressed by Kuntz. ${ }^{13}$ He puts forward cogent arguments to support the concept that those trials and registries that show an adverse outcome in diabetic patients treated by multivessel PCI have been those that have included patients with the most severe disease, who are at greatest risk of late coronary occlusive events. When diabetic patients with less extensive disease are studied, the outcomes of treatment by PCI or CABG are similar. This may explain why both treatment strategies were associated with similar mortality in the randomised intervention treatment of angina (RITA-1) trial, ${ }^{14}$ which included patients with much less extensive disease ( $45 \%$ had single vessel disease). The BARI registry also failed to show a differential survival effect. ${ }^{15}$ Unlike the BARI trial, where randomisation ensured a balance of baseline characteristics, in the BARI registry the incidence of three vessel disease was almost twice as high in the surgical group compared with the PCI group. Thus patients at higher risk of later coronary occlusive events were more likely to be given the protection of bypass grafts.

\section{INTIMAL REMODELLING THERAPY}

The arguments above would suggest that the introduction of stents that prevent restenosis, and glycoprotein IIb/IIIa receptor antagonists to reduce periprocedural myocyte necrosis, will have little impact on medium and long term outcome of multivessel PCI in diabetics. Perhaps we need a radically different approach. Following successful PCI, the atheromatous arterial wall is re-lined by smooth muscle cells and intracellular matrix, with a very low risk of acute luminal obstruction after six months. ${ }^{16}{ }^{17}$ If drug eluting stents live up to their early promise to abolish restenosis, then instead of "spot" therapy to treat angiographically defined culprit lesions, perhaps we should dilate and stent the entire proximal 6-7 cm of all major epicardial vessels that show disease (defined angiographically and/or by intravascular ultrasound). Such a strategy might be termed "intimal remodelling therapy". This proposal is a logical extension of the concept of focal plaque sealing, which was put forward by Meier at a time when the burden of repeat revascularisation caused by restenosis might have obviated any benefit. ${ }^{18}$

Perhaps we are now entering an era where the aim will be to treat as much of the coronary artery as possible, rather than to target only short segments for fear of restenosis. Clearly there are specific conditions that would need to be met before such a strategy could be adopted. We would need to be convinced that the medium to long term stability of neointima created by PCI using a long drug eluting stent is similar to that generated by conventional focal PCI. Treating long segments, particularly of the left anterior descending artery, would risk jailing side branches. We would therefore need more data to support the early reports of side branch preservation with drug eluting stents. We then might consider a prospective randomised trial to compare "intimal remodelling therapy" with CABG in diabetic patients with multivessel disease, particularly in those patients suitable for left anterior descending revascularisation by both techniques. We might then be able to demonstrate that intimal remodelling therapy by PCI can alter the natural history of disease progression, and be able to improve the otherwise poor prognosis of diabetic patients with severe diffuse multivessel disease.

\section{REFERENCES}

1 Duncan C, Chalmers J, Campbell IW, et al. An audit of non-insulin-dependent diabetics attending a district general hospital diabetic clinic: implications for shared care between hospital and general practice. Health Bulletin 1992;50:302-8.

2 Laing SP, Swerdlow AJ, Slater SD, et al. The British Diabetic Association cohort study, II: cause-specific mortality in patients with insulin-treated diabetes mellitus. Diabet Med 1999:16:466-71.

3 Amos AF, McCarty DJ, Zimmet P. The rising global burden of diabetes and its complications: estimates and projections to the year 2010. Diabet Med 1997;14(suppl 5):S1-85.

4 The Bypass Angioplasty Revascularization Investigation (BARI) Investigators. Comparison of coronary bypass surgery with angioplasty in patients with multivessel disease. The bypass angioplasty revascularization investigation (BARI) investigators. [erratum appears in $\mathrm{N}$ Engl J Med 1997;336:147]. N Engl J Med 1996;335:217-25.

5 Mueller HS, Cohen LS, Braunwald E, et al. Predictors of early morbidity and mortality after thrombolytic therapy of acute myocardial infarction. Analyses of patient subgroups in the Thrombolysis in myocardial infarction (TIMI) trial, phase II. Circulation 1992;85:1254-64

6 The Bypass Angioplasty Revascularization Investigation (BARI) Investigators. Influence of diabetes on 5-year mortality and morbidity in a randomized trial comparing CABG and PTCA in patients with multivessel disease: the bypass angioplasty revascularization investigation (BARI). Circulation 1997;96:1761-9.

7 The Bypass Angioplasty Revascularization Investigation (BARI) Investigators. Seven-year outcome in the bypass angioplasty revascularization investigation (BARI) by treatment and diabetic status. J Am Coll Cardiol 2000;35: $1122-9$. 
8 King SB, Kosinski AS, Guyton RA, et al. Eight-year mortality in the Emory angioplasty versus surgery trial (EAST). J Am Coll Cardiol 2000;35: $1116-21$

9 Kurbaan AS, Bowker TJ, Ilsley CD, et al. Difference in the mortality of the CABRI diabetic and nondiabetic populations and its relation to coronary artery disease and the revascularization mode. Am J Cardiol 2001;87:947-50.

10 Serruys PW, Unger F, Sousa JE, et al. Comparison of coronary-artery bypass surgery and stenting for the treatment of multivessel disease. $N$ Engl J Med 2001;344:1117-24.

11 Abizaid A, Costa MA, Centemero M, et al. Clinical and economic impact of diabetes mellitus on percutaneous and surgical treatment of multivessel coronary disease patients: insights from the arterial revascularization therapy study (ARTS) trial. Circulation 2001;104:533-8.

12 Serruys PW. Coronary-artery bypass surgery versus stenting for multivessel disease [letter]. N Engl J Med 200 1;345:1642-3.

13 Kuntz RE. Importance of considering atherosclerosis progression when choosing a coronary revascularization strategy: the diabetes-percutaneous transluminal coronary angioplasty dilemma Circulation 1999;99:847-51.

14 Henderson RA, Pocock SJ, Sharp SJ, et al. Long-term results of RITA-1 trial: clinical and cost comparisons of coronary angioplasty and coronary-artery bypass grafting. Randomised intervention treatment of angina. Lancet 1998;352:1419-25.

15 Detre KM, Guo P, Holubkov R, et al. Coronary revascularization in diabetic patients: a comparison of the randomized and observational components of the bypass angioplasty revascularization investigation (BARI). Circulation 1999:99:633-40.

16 Kober G, Vallbracht C, Kadel C, et al. Results of repeat angiography up to eight years following percutaneous transluminal angioplasty. Eur Heart J 1989;10(suppl G):49-53.

17 Kitazume H, Kubo I, Iwama T, et al. Long-term angiographic follow-up of lesions patent 6 months after percutaneous coronary angioplasty. Am Heart J 1995;129:441-4.

18 Meier B, Ramamurthy S. Plaque sealing by coronary angioplasty. Cathet Cardiovasc Diagn 1995;36:295-7.

\section{IMAGES IN CARDIOLOGY}

\section{Difficult haemostasis following diagnostic transfemoral angiogram caused by inadvertent} puncture of the arteria profunda femoris

$\mathrm{D}$

uring transfemoral catheterisation one must be careful not to make the arterial puncture far below the inguinal ligament distal to the origin of the branches of the femoral artery. We illustrate a case of inadvertent puncture of the profunda femoris artery during diagnostic cardiac catheterisation. After the sheath was taken out full haemostasis could not be achieved despite prolonged manual pressure. Doppler ultrasound of the groin revealed a complex structure within the right groin consistent with an overlying haematoma. It was difficult to visualise the underlying native vessels and to help surgical planning a computed tomographic (CT) angiogram was done. This coronal section of the CT angiogram clearly shows a complex bi-lobed pseudoaneurysm arising directly from the right profunda femoris artery, separate from the right superficial femoral artery. There is an organising haematoma superficial to the pseudoaneurysm. Surgical repair of the puncture hole was successfully undertaken.

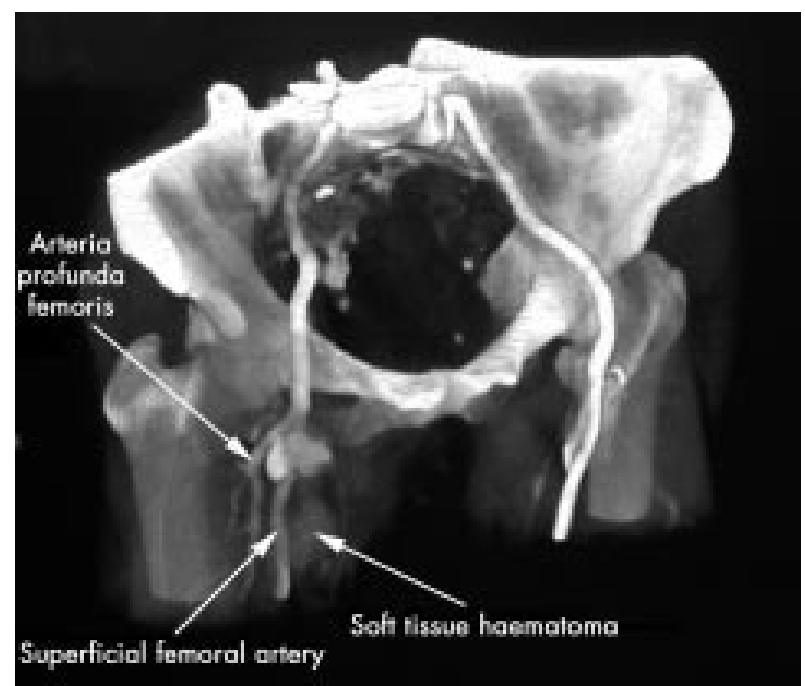

\section{STAMPS IN CARDIOLOGY}

\section{Atropa belladonna}

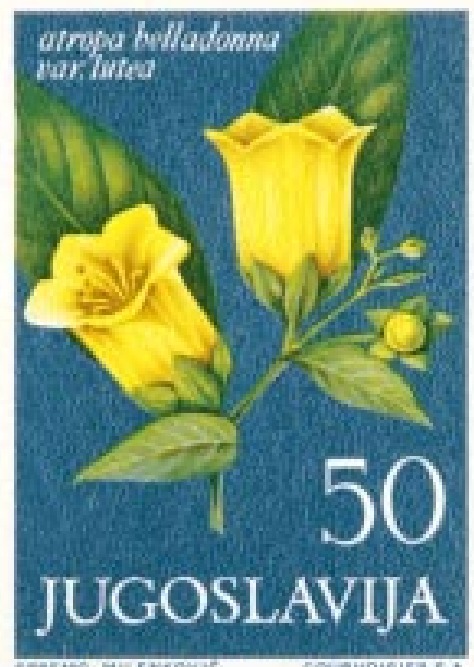

Atropa belladonna is deadly nightshade, a very poisonous plant named by Linnaeus after one of the Fates, Atropus, who cut the thread of life. Atropine is obtained from its leaf and berries and was first isolated in 1831. In 1867 von Bezold showed that it blocked the cardiac effects of vagal stimulation. Sir James Mackenzie (1853-1925) showed that it would revert partial but not complete heart block. He studied its effect on heart rate in digitalised patients in atrial fibrillation and submitted a paper on this subject to Heart. It was rejected by the editor, Sir Thomas Lewis, and the infuriated Mackenzie replied, "You might as well put on the forefront of the journal 'No articles will be accepted which are not in accordance with the (temporary) beliefs of the Editor'". It was hardly used in cardiology until the introduction of coronary care units led to its use for the treatment of bradycardia and heart block after myocardial infarction.

Atropa belladonna has appeared on a number of stamps from different countries over the years The one chosen for illustration comes from Yugoslavia in 1965 and was part of a set of six stamps issued depicting medicinal plants. Peppermint and the rusty foxglove were the two highest values in the set.

M K Davies

A Hollman 